\title{
O USO DE VENTILADORES NA PANDEMIA DO COVID-19
}

\author{
Laura Diehl Barbosa ${ }^{1}$ \\ Grazielle Zanatta ${ }^{1}$ \\ Edmara Laura Campiolo ${ }^{2}$
}

\section{RESUMO}

Introdução: A ventilação mecânica é realizada em fases graves do COVID-19, em que há uma deterioração da respiração do paciente e evolução para síndrome da angústia respiratória aguda (SARA). Objetivo: Demonstrar os desafios enfrentados pelos países em relação ao uso dos ventiladores na pandemia. Métodos: Estudo realizado por meio do levantamento bibliográfico em revistas científicas e plataformas de dados online. Resultados: Os desafios do uso da ventilação mecânica são: falha no distanciamento social, testes diagnósticos demorados e ineficazes, quantidade e distribuição assimétrica de leitos de unidade de terapia intensiva (UTI) e dilemas bioéticos sobre quem deve ter acesso aos ventiladores.

Conclusão: Esses obstáculos aumentam o número de infectados e reduzem a disponibilidade de ventiladores, podendo colapsar sistemas de saúde no mundo, especialmente no Brasil. A partir disso, surgem novas alternativas para que a demanda desse recurso seja sanada.

Palavras-chave: COVID-19, insuficiência respiratória, ventilação mecânica, ventiladores, terapia intensiva

\section{ABSTRACT}

Introduction: The use of ventilators is highly recommended in advanced phases of COVID-19, when patients present deterioration of lung function and acute respiratory failure. Objective: To present the challenges faced by many countries on the use of ventilators during pandemic. Methods: This study was based on bibliographic research on cientific magazines and online database. Results: The challenges facing mechanical ventilation are: failure on implementing social distancing, lack of diagnosis and late diagnosis, unequal distribution of intensive care units and bioethical issues among who gets access to intensive care. Conclusions: The shortage of ventilators and high number of people infected with COVID-19 could bring health systems to collapse in many countries, especially Brazil. Therefore, new alternatives are being studied in order to solve the presented issue.

Keywords: COVID-19, respiratory insufficiency, mechanical ventilation, ventilators, intensive care

\footnotetext{
${ }^{1}$ Acadêmico de Medicina da Pontifícia Universidade Católica do Paraná - campus Londrina. Laura Diehl Barbosa - Av. Jockei Club, 485 - Hipica, Londrina - PR, 86072-360

${ }^{2}$ Preceptora de Pediatria da Pontifícia Universidade Católica do Paraná - campus Londrina, PR, Brasil. E-mail: edmaralaura@hotmail.com
}

Como citar este artigo / How to cite this article

Barbosa LD, Zanatta G, Campiolo EL. O uso de ventiladores na pandemia do COVID-19 The use of ventilators in COVID-19 pandemic Uso de ventiladores na pandemia. Interam J Med Health 2020;3:e202003052. 


\section{INTRODUÇÃO}

No ano de 2019, o número de pneumonias causadas pelo coronavírus cresceu substancialmente na região de Wuhan, na China. Em pouco tempo, instalou-se a pandemia da nova doença elencada pela Organização pandemia da nova doença elencada pela Organização
Mundial da Saúde (OMS) como COVID-19, acrônimo referente a "Coronavirus disease-2019". A infecção pelo coronavírus tipo 2 da síndrome respiratória aguda grave (SARS-CoV-2), patógeno do COVID-19, provocou a internação de muitos pacientes pelo desenvolvimento de síndromes respiratórias e aumentou o uso da ventilação mecânica. Esse súbito aumento da demanda por leitos ventiladores abalou, em muitos páses, infraestrutura na área da saúde [1]

A ventilação mecânica consiste na substituição completa ou parcial da atividade ventilatória espontânea do paciente que apresenta insuficiência respiratória aguda ou crônica agudizada. O uso dos ventiladores promove melhor desempenho de trocas gasosas e diminui o trabalho respiratório, podendo ser não invasivo, por meio de uma interface externa, como máscara através de intubação através de intubaçaco ou traqueostomia [2]. Estima-se que $20 \%$ dos pacientes diagnosticados com COVID-19 desenvolvem a forma grave da doença, podendo adquirir a síndrome da angústia respiratória aguda (SARA), a complicação mais severa. A partir disso, o paciente acaba manifestando uma insuficiência respiratória hipoxêmica aguda e cerca de 42 a 100\% desses pacientes necessitam de auxílio respiratório [3].

Além disso, os pacientes com Saturação de Oxigênio (SpO2) $\leq 93 \%$ (Kim e Gandhi, 2020) ou com rápida progressão da doença ou com sinais de angústia respiratória devem ser entubados e ventilados por duas semanas ou mais [3].

Essas dificuldades permeiam diversos países, nos Estados Unidos há cerca de 60 mil a 160 mil ventiladores, não sendo um número suficiente para 0 combate da doença [5], visto que muitas outras doenças crônicas necessitam do suporte ventilatório, gerando incongruências quanto ao uso do equipamento [3]

No Reino Unido, estima-se que dois terços dos pacientes com COVID-19 necessitem de ventilação já nas primeiras 24 horas6 e, na Itália, 10 a 25\% dos pacientes hospitalizados requerem esse suporte [7]. Já os países do sul asiático, como Índia, Paquistão e Bangladesh, também têm apresentado dificuldades quanto ao uso de ventiladores, em especial, os sul asiáticos apresentam, ainda, poucos hospitais capazes de isolar pacientes $e$ fornecer o suporte respiratório, expandindo a questão da falta de ventiladores à falta de recursos extras para a execução do serviço [8].

No Brasil, segundo o Ministério da Saúde, existem por volta de 65 mil respiradores e aproximadamente 46 mil estão disponíveis no Sistema Único de Saúde (SUS) [9]. Já o número de leitos de UTI no país é estimado em 2,03 a cada 10 mil habitantes, com variação nas diferentes regiões brasileiras, sendo que $72 \%$ das regiōes de saúde possuem déficit nesse quesito [10].

Diante da pandemia do COVID-19, torna-se relevante analisar os dados envolvendo o uso de ventiladores para os desafios estruturais e éticos enfrentados pelos países, com enfoque no Brasil, para tratar os pacientes que desenvolvem insuficiência respiratória e necessitam de suporte ventilatório nos leitos de UTI.

\section{METODOLOGIA}

O presente estudo consiste em uma revisão integrativa de literatura Para a realização deste trabalho, foi desempenhada busca manual em periódicos e órgãos internacionais e nacionais de grande impacto, como: The Lancet, New England Journal of Medicine, The BMJ, Organização Mundial da Saúde, Ministério da Saúde do Brasil e a plataforma digital do DATASUS, além de busca automática em bases de dados internacionais como UpToDate, PubMed eGoogle Scholar.

Foram utilizados, para busca dos artigos, os seguintes descritores e suas combinações nas línguas portuguesa e inglesa: "ventilação mecânica", "ventiladores no COVID-19", "insuficiência respiratória no COVID-19", "vagas de UTI na pandemia", "severe acute respiratory syndrome", "acute respiratory failure" e "ventilation during pandemic", sendo selecionadas 30 publicações.

Os critérios de inclusão utilizados foram trabalhos publicados em português e inglês que possuíssem relevância para o estudo desenvolvido. Já os critérios de exclusão foram baseados nos trabalhos que não apresentavam esses idiomas e que não demonstravam relevância ao estudo, ou com vieses que comprometessem os resultados.

\section{RESULTADO}

Tendo em vista que a doença grave causada pelo COVID-19 preenche os critérios de ventilação mecânica e que esta se desenvolve em aproximadamente $14 \%$ dos pacientes hospitalizados, com quadro característico de síndrome da angústia respiratória aguda (SARA) ou até mesmo insuficiência respiratória hipoxêmica aguda, os desafios que limitci os desafios que limitam o uso desse recurso estão diretamente ligados a disequilbrio entre a porcentagem de pacientes que necessitam da ventilação e a quantidade
de ventiladores disponíveis.

Foi possível visualizar que esses desafios têm origem pré hospitalar, mais especificamente no número elevado de pessoas infectadas. Dessa forma, o distanciamento social consiste em importante ferramenta para minimizar - contágio da população. Verificou-se, também, que - contágio da popuasa. Veificou-se, tambem, a a realizaçáo de testes diagnósticos rápidos e eficazes podem diminuir o índice de intubações, haja vista que, a partir deles, é possível encaminhar pessoas com doenças pulmonares crônicas para uma ventilação não invasiva, disponibilizando o método invasivo para os pacientes diagnosticados com COVID-19, restringindo, ainda, as lesões decorrentes da intubação.

As Unidades de Terapia Intensiva (UTI) também foram apresentadas como um outro obstáculo no combate da doença, considerando que os leitos de UTI, no Brasil, em especial, são distribuídos de maneira não uniforme por todo território nacional, além de serem escassos quando relacionados à demanda de pacientes na pandemia. Ademais, foi observado que os dilemas bioéticos são frequentes quando se é discutido quem deve ou não receber o suporte respiratório, uma vez que a equipe médica deve decidir, com base no estado do paciente, se a ventilação lhe será útil ou se outra pessoa terá maiores benefícios com ela.

Por fim, o número de ventiladores é um dos grandes fatores limitantes do tratamento da doença, sendo constatado déficit desse recurso no Brasil e no mundo, fazendo com que alternativas fossem criadas para suprir essa necessidade, como a produção de novos ventiladores por montadoras e a ventilação de diversos pacientes com um único ventilador, ainda sub testada e não regulamentada.

\section{DISCUSSÃO}

\section{A ventilação mecânica no CoVID-19}

Quem necessita da ventilação mecânica?

De acordo com o guia chinês, a doença do COVID-19 pode ser classificada conforme sua gravidade: leve, moderada, grave e seu estado crítico, em que cada uma apresenta um quadro e um manejo clínico diferente, sendo que o suporte ventilatório é necessário a partir da fase grave da doença, caracterizada pela angústia respiratória 11]. Segundo a Organização Mundial da Saúde, 14\% dos pacientes acabam desenvolvendo esta forma mais grave da doença, sendo que $5 \%$ necessita de cuidados nas Unidades de Terapia Intensiva (UTI), com uma média de permanência de três a quatro semanas [12].

O quadro grave se adequa nos critérios de ventilação pois o paciente passa a apresentar hipóxia (saturação de oxigênio $\leq 93 \%$ ), taquipnéia ( $\geq 30$ respirações $/ \mathrm{min}$ ) ou insuficiência respiratória e comprometimento maior que $50 \%$ do parênquima pulmonar nos exames de imagen [4]. Já no estágio crítico da doença, segundo o guia chinês, é possível visualizar a síndrome da angústia respiratória aguda (SARA), podendo ocorrer até mesmo choque e insuficiência de outros órgãos [11], estima-se que 30 a $100 \%$ dos pacientes nesta fase requerem ventilação mecânica [3].

Um estudo descrito nas UTIs dos hospitais de Seattle, entre os dias 24 de fevereiro a 9 de março, relatou o uso de ventilação mecânica em $75 \%$ dos pacientes cominsuficiência respiratória hipoxêmica aguda confirmados para COVID-19, uma complicação da síndrome da angústia respiratória aguda13. Em Wuhan, um estudo relatou o aparecimento de SARA em $20 \%$ dos pacientes, sendo que $12,3 \%$ destes careceram de suporte ventilatório [1]

As diretrizes do Ministério da Saúde para o tratamento do COVID-19 sugerem a intubação endotraqueal para pacientes graves, que não obtiveram alívio dos sintomas (desconforto respiratório persistente e/ou hipoxemia) após oxigenoterapia padrão, pacientes cujos sintomas (dificuldade respiratória, frequência respiratória $>30 / \mathrm{min}$, indice de oxigenação $\mathrm{PaO} / / \mathrm{FiO}^{2}<150 \mathrm{mmHg}$ ) persistem ou exacerbam após oxigenação nasal de alto fluxo (HFNO) ou ventilação não invasiva por 2 horas [14].

Para os pacientes com leve desconforto respiratório, recomenda-se o uso de cateter nasal com baixo fluxo de oxigênio [15]. A ventilação mecânica não invasiva pode ser realizada quando a cânula nasal e oxigenoterapia com máscara forem ineficazes, no entanto, é necessário seguir um protocolo para evitar contaminação dos profissionais de saúde que realizam esse tipo de ventilação [14].

Desafios da ventilação nos pacientes com COVID-19

De maneira geral, os procedimentos de intubação consistem na pré oxigenação do paciente com uma fração 
inspirada de oxigênio (FiO2) 100\%, abertura das vias aéreas, aplicação de anestesia e, por fim, passagem do tubo endotraqueal [16] se complicada devido à urgência do procedimento, que dificulta a análise prévia das vias aéreas e devido ao risco de infecção cruzada dos profissionais que realizam o procedimento. O uso de máscara N95, cobertura corporal fluido-resistente, duas camadas de luvas, óculos, protetor facial e protetores de sapato fluido-resistentes dificulta a realizaçăo de movimentos finos e precisos, os quais a intubação demanda [16].

Além disso, os pacientes com síndrome respiratória aguda hipoxêmica apresentam pouca reserva respiratória e falha dos mecanismos compensatórios pulmonares. Muitos pacientes com SpO2 baixa, quando intubados, podem apresentar imediata queda da $\mathrm{SpO} 2$ e gradual elevação com auxílio de máscaras de ventilação manual. Em Wuhan, para melhor performance da intubação nos pacientes com COVID-19, a tarefa foi direcionada aos profissionais com maior experiência clínica [16]

Existe, ainda, o risco dos pacientes desenvolverem "lesão pulmonar induzida pela ventilação mecânica" [16] A má regulação dos aparelhos de ventilação pode provocar deformação do epitélio alveolar e perda da barreira epitelial, com aumento de mediadores inflamatórios no pulmão e bactérias na circulação sanguínea [17].

\section{Disponibilidade de recursos na pandemia}

Panorama da terapia intensiva e ventiladores no Brasil

Segundo atualização da Associação de Medicina
Nos pacientes com COVID-19, a intubação torna-

Intensiva Brasileira (AMIB) de Março de 2020, o Brasil possui 45.848 leitos de Unidade de Terapia Intensiva (UTI) sendo 22.844 do Sistema Único de Saúde (SUS) e 23.004 no sistema privado 18 A obrigatoriedade de leitos de UTI todos os municipios brasileiros, apenas [19].

Tendo como base o censo de 2016 da AMIB, os leitos UTI dividem-se desigualmente por região brasileira, sendo que o Sudeste apresenta a maior concentração, com $53.4 \%$ do total de leitos UTI no Brasil. Em seguida, vem a região Nordeste com $18,3 \%$, a região Sul com $14,6 \%$, 0 Centro-Oeste com $8,4 \%$ e o Norte com $5,2 \%$. Em valores absolutos, a região Sudeste possui uma média de 23 leitos UTI por estabelecimento, o Nordeste possui uma média de 19 leitos, o Norte 17 leitos e o Centro-Oeste e a região Sul, 21 leitos [19].

A média de leitos UTI correspondia a 2,03 para cada 10 mil habitantes. No entanto, há divergências quando se compara o número de leitos de UTI disponíveis nas diferentes regiões brasileiras, os leitos disponíveis pelo SUS e rede privada e nas capitais e interior, representado na Tabela 1

Na região Sul, por exemplo, a média total é de 2,07 leitos UTI para cada 10 mil habitantes, no entanto na capital a média sobe para 4,79 e no interior cai para 1,66. Da mesma forma, a média de leitos UTI disponíveis pelo de Saúde (SUS) na região Sul corresponde a 1,71 e os leitos UTI não SUS correspondem a 3,23 para cada 10 mil habitantes [19].

Tabela 1. (Proporção de leitos de UTI por 10.000 habitantes): Censo da AMIB de 2016 mostra proporção de leitos de UTI por 10 mil habitantes no Brasil.

\begin{tabular}{|l|c|c|c|c|c|c|c|c|c|}
\cline { 2 - 12 } \multicolumn{1}{c|}{} & \multicolumn{3}{c|}{ Capital } & \multicolumn{3}{c|}{ Interior } & \multicolumn{3}{c|}{ Total } \\
\hline Região & SUS & Não SUS & Total & SUS & Não SUS & Total & SUS & Não SUS & Total \\
\hline Centro-Oeste & 2,51 & 7,9 & $\mathbf{4 , 1 8}$ & 0,54 & 4,74 & $\mathbf{1 , 0 7}$ & 1,17 & 6,61 & $\mathbf{2 , 2 4}$ \\
\hline Nordeste & 3,22 & 5,7 & $\mathbf{4 , 0 2}$ & 0,4 & 3,83 & $\mathbf{0 , 6}$ & 0,88 & 4,97 & $\mathbf{1 , 3 5}$ \\
\hline Norte & 1,9 & 7,22 & $\mathbf{3 , 0 7}$ & 0,32 & 2,09 & $\mathbf{0 , 4}$ & 0,75 & 5,64 & $\mathbf{1 , 2 3}$ \\
\hline Sudeste & 3,27 & 6 & $\mathbf{4 , 6 4}$ & 1,32 & 3,35 & $\mathbf{1 , 9 1}$ & 1,69 & 4,32 & $\mathbf{2 , 5 8}$ \\
\hline Sul & 5,11 & 4,45 & $\mathbf{4 , 7 9}$ & 1,38 & 2,79 & $\mathbf{1 , 6 6}$ & 1,71 & 3,23 & $\mathbf{2 , 0 7}$ \\
\hline Média Brasil & 3,07 & 6,04 & $\mathbf{4 , 2 6}$ & 0,89 & 3,33 & $\mathbf{1 , 3 3}$ & 1,3 & 4,45 & $\mathbf{2 , 0 3}$ \\
\hline
\end{tabular}

Fonte: Associação de Medicina Intensiva Brasileira [19]
A atualização de Março de 2020 da AMIB mostra um panorama semelhante ao de 2016, sendo que Brasil, atualmente, apresenta 2,2 leitos de UTI por $10 \mathrm{mil}$ habitantes. Por região, são 2,7 leitos 10 mil habitantes no Sudeste, 2,5 leitos/10 mil habitantes no Centro-Oeste, 2,2 leitos/10 mil habitantes no Sul e 1,5 leito/10 mil habitantes no Nordeste. No Norte são 0,90 leito/10 mil habitantes pelo SUS e 4,7 leitos $/ 10$ mil habitantes pela rede particular [18.

Segundo o Cadastro Nacional de Estabelecimento de Saúde (CNES) de Abril de 2020, o Brasil possui 70.516 equipamentos de ventilação mecânica: 36.264 na região Sudeste, 13.812 no Nordeste, 10.074 no Sul, 6.539 no Centro-Oeste e 3.827 na reg nacordo com o Ministerio da Savde, 46 mil ventiladores estão disponíveis no SUS [9].

O Instituto de Estudos para Políticas de Saúde (IEPS) apontou para uma defasagem de equipamentos em 316 das 438 regiões de saúde presentes no Brasil, o que corresponde a $61 \%$ da população suprida unicamente pelo SUS, A região de saúde do Rio de Janeiro, por

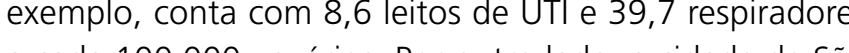
a cada 100.000 usabilos. Por outro lado, a cidade de São Paulo conta com 18,6 leitos de UTI e 55,5 respiradores por 100.000 usuários [10].

O IEPS realizou também uma estimativa da taxa de infecção populacional necessária para a ocupação de $100 \%$ dos leitos UTI e os resultados mostraram que para metade das regionais de saúde, uma taxa de infeccão de $9 \%$ dos habitantes seria suficiente para ocupar 100\% dos leitos de UTI e para um quarto das regionais, 5,6\% de infectados bastaria para sobrecarregar o sistema [10]

O Ministério da Saúde (MS), em resolução de 2010, preconiza a presença de um ventilador pulmonar mecânico microprocessado a cada 2 leitos de UTI adulto, com uma reserva operacional a cada 5 leitos. A mesma resolução prevê um equipamento para ventilação pulmonar mecânica não invasiva a cada 10 leitos, quando o ventilador pulmonar mecânico microprocessado não possuir recursos para realizar a modalidade de ventilação não invasiva. Além disso, a UTI adulto deve conter um material de interface facial para ventilação pulmonar não invasiva para cada 5 leitos [20].

Diante da crise do novo coronavírus, o Ministério da Saúde investiu R\$658,5 milhões em 14.100 novos respiradores pulmonares produzidos por empresas nacionais para a assistência de pacientes graves atendidos na rede pública de saúde [9].
Panorama da ventilação mecânica no mundo

Durante a pandemia do COVID-19 muitos desafios podem ser descritos, desde a escassez de testes diagnósticos, ventiladores e leitos de UTI até a dificuldade o distanciamento social instaurado em diversos países como possível solução para o achatamento da curva de novas infecções é um importante fator quando o uso de ventiladores está em pauta, já que esses equipamentos são limitados [5]. Nos países sul asiáticos, por exemplo, esse tem sido um grande desafio, já que a implementação da quarentená dificultada pela incapacidade de trabahar . Considerando os testes diagnósticos como um
Cucão e a crise econômica gerada pela pandemia [8]. outro empecilho quanto ao uso de suporte ventilatório, é necessário ter em vista que pacientes devidamente diagnosticados podem receber uma ventilação adequada, evitando que pacientes com doenças crônicas, não relacionadas com o COVID-19, sejam entubados enquanto esperam os resultados do teste, podendo receber uma os danosa, deixando com que esse método ventilatório possa estar disponívelàqueles com a forma grave da doença viral [5].

Já a escassez de ventiladores pode ser elucidada com a estimativa do centro de controle e prevenção de doenças que calcula uma margem de 1,4 a 31 pacientes por ventilador nos Estados Unidos, esse é um grande desafio enfrentado na pandemia, envolvendo, ainda, fatores éticos [7].

Dilemas bioéticos da ventilação mecânica e novas alternativas

Dilemas bioéticos

A decisão de intubar o paciente deve ponderar os riscos e benefícios do procedimento e, acima de tudo, deve ser tomada rapidamente, pois enquadra uma situação de vida ou morte. Diante do quadro de urgência da intubação e escassez de equipamentos na pandemia, foram instauradas triagens dos pacientes para decidir quem tem prioridade no acesso aos leitos de UTI e à ventilação mecânica. Apesar de serem decisões difíceis, as triagens devem ser encaradas como possibilidade de salvar vidas e não como sentenças de morte [7].

A triagem para intubação do paciente é realizada em três etapas: aplicação de critérios de exclusão, como 
situações de saúde irreversíves (choque irreversível) e Intensiva e colapso no sistema de saúde. Para isso, deve avaliação da severidade da doença por meio da pontuação ser feita ampliação dos testes diagnósticos do COVID-19 no Sequential Organ Failure Assessment (SOFA) [7]. nos pacientes com suspeita e os critérios para realização nutricional e cognitivo, os desejos do paciente e o número realização rápida e eficaz de ventiladores disponíveis no local [21].

A idade é um dos principais fatores de ponderação nas triagens. Pacientes jovens geralmente são priorizados na fila do acesso à terapia intensiva, pois apresentam maior responsividade à ventilação mecânica, enquanto os pacientes mais idosos são menos responsivos e possuem uma certa deteriorização da função pulmonar inerente à idade. Além disso, entende-se que os mais novos deveriam ter acesso prioritário à terapia intensiva por terem vivido menos, enquanto os pacientes mais velhos possuem uma trajetória que já foi por mais tempo percorrida [22].

Os autores que não concordam com a priorização dos mais jovens no tratamento intensivo, clamam que a decisão corrobora para a disseminação do preconceito contra os mais velhos [22].

Na França, foram criados comitês de suporte ético, com profissionais de saúde de diferentes áreas da medicina, para auxiliar na tomada de decisão dos médicos e promover suporte psicológico e científico em tempos de pandemia [21]. Em Nova York, tem sido realizado racionamento dos ventiladores, com avaliação constante do paciente para ser decidido se ele deve ser mantido no suporte ventilatório ou se o equipamento pode ser disponibilizado para outro paciente [7].

Por fim, destaca-se a importância de manter uma boa comunicação com a família dos pacientes, explicando a lógica das triagens e a ordem de não intubação para pacientes com alto risco de deterioraçãa. Os comitês de ética devem estar à frente dessa comunicação para que a responsabilidade da escolha conjunta não recaia sobre um profissional de saúde isoladamente. Além disso, uma vez que a decisão de desligamento da ventilação de um paciente é feita, deve ser garantido a ele o melhor atendimento, por meio de profissionais com experiência em cuidados paliativos e promoção de suporte emocional à familia [7]

Alternativas para as dificuldades enfrentadas pandemia

Diante da escassez de recursos ao qual a pandemia expõe, a classificação dos pacientes de acordo com a doença apresentada auxilia no encaminhamento hospitalar e evita a superlotação de Unidades de Terapia pacientes que poderiam recuperarse ambulatoriasmente Cingapura destacou-se nesse quesito, já que desde o início do surto rastreou rigorosamente todos os contatos de casos, utilizou testes generalizados para ainda manteve uma comunicação transparente com a população [24].

Ainda sobre o rastreamento precoce da doença, em Wuhan, estudos apontaram algumas recomendações para o controle da pandemia, como o desenvolvimento de modelos matemáticos que previssem o número de pacientes e a quantidade de equipamentos necessários para os casos, além da identificação de biomarcadores importantes que precedem uma doença grave, como altas auxiliaria no preparo de um futuro suporte respiratório [25]. descrevem três vias de ação para reduzir o desequilíbrio via seria o achatamento da curva pelo distanciamento social, seguido de uma transferência de ventiladores de diversos locais do país de acordo com as projeções epidemiológica picos assíncronos, e por fim o aumento na produção dos aparelhos [26].

A companhia de tecnologia britânica, Dyson Ltd, desenvolveu um novo ventilador chamado "CoVent", que atende aos padrões da especificação clínica27. Outra alternativa que poderia suprir a necessidade desse suporte, ainda sub-testada e não regulamentada, é a possibilidade de ventilar diversos pacientes com quadro pulmonar similar urilizando um único ventilador. Os pacientes ficariam expostos a um mesmo circuito dinâmico, o que, no entanto, poderia gerar complicações, como infecção cruzada por troca gasosa dos pacientes e incapacidade oxigênio [28].

Além disso, no caso de uma parada cardíaca, a ventilação de todos os pacientes precisaria ser interrompida para que o procedimento com balão fosse iniciado. Assim, existem questões éticas a serem discutidas sobre o compartilhamento de ventiladores [29]. pacientes que poderiam recuperar-se ambulatorialmente os pacientes suspeitos, iniciou uma quarentena precoce quantidades de proteína $C$ reativa e linfopenia, o que

Estudos realizados nos Estados Unidos, ainda, entre a oferta e a demanda de ventiladores: a primeira de definir o volume corrente individual e concentração de
É importante ressaltar que os ventiladores mecânicos não são usados somente nos casos de COVID-19, mas também em pacientes com doenças pulmonares crônicas, esclerose lateral amiotrófica, acidentes vasculares cerebrais e miastenia grave, o que torna a demanda pelos aparelhos ventilatórios ainda mais alta [30]0.

\section{CONCLUSÃO}

A partir do que foi apresentado, concluímos que há um déficit infra-estrutural no Brasil e no mundo, relacionado ao número de ventiladores disponíveis no combate ao COVID-19, o que gera dilemas bioéticos a respeito do uso desses aparelhos. $\mathrm{Em}$ certas regiões brasileiras, especialmente, foi visto que o déficit na medicina intensiva já se fazia presente antes da déficit na medicina intensiva já se fazia presente antes da
instalação da pandemia. O número crescente de infectados instalação da pandemia. O número crescente de infectados pelo novo coronavírus eleva a quantidade de pacientes que desenvolverão a forma grave da doença, com necessidade para o traça mecânica. Assim, fatores que pom necessidade maior eficácia dos testes diagnósticos e a produção de novos maior eficácia d

\section{REFERÊNCIAS}

1. Mclntosh, K. Coronavirus disease 2019 (COVID-19): Epidemiology, virology clinical features, diagnosis, and prevention. UpToDate. Abril de

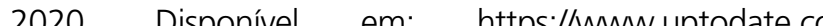
contents/coronavirus-disease-2019-covid-19epidemiology-virology-clinical-features-diagnosis-andprevention?search =ventilators $\% 20$ in $\% 20$ COVID $-19 \% 20$ pandemic\&source $=$ search_result\&selectedTitle $=5 \sim 150$ \&usage_type=default\&display_rank=5\#H3504376427.

2. Hyzy, R. C.; McSparron, J. I. Noninvasive ventilation in adults with acute respiratory failure: Practical aspects of initiation. UpToDate. Abril de 2020. Disponivel em https://www. uptodate.com/contents/noninvasiveventilation-in-adults-with-acute-respiratory-failurepractical-aspects-of-initiation?search=noninvasive $\% 20$ ventilation\&source $=$ search_result\&selectedTitle $=1 \sim 150 \&$ usage_type $=$ default $\&$ display_rank $=1$

3. Anesi, G. L. Coronavirus disease 2019 (COVID-19): Critical care and airway management issues. UpToDate. Abril de 2020. Disponível em: https://www.uptodate. com/contents/coronavirus-disease-2019-covid-19-critical-
care-and-airway-management-issues\#H4080233186.

4. Kim, A. Y.; Gandhi, R. T. Coronavirus disease 2019 (COVID-19): Management in hospitalized adults. UpToDate. Abril de 2020. Disponível em: https://www. uptodate.com/contents/coronavirusdisease-2019-covid-19-management-in-hospitalizedadults? search=ventilators $\% 20 \mathrm{in} \% 20 \mathrm{COVID}-19 \% 20$ pandemic\&source $=$ search_result\&selectedTitle $=7 \sim 150$ \&usage_type=default\&display_rank=7\#H2294322673. 5. Ranney, M. L.; Griffeth, V.: Jha, A. K. Critical Supply Shortages - The Need for Ventilators and Personal Protective Equipment during the Covid-19 Pandemic. N Engl J Med [Internet]. 30 de Abril de 2020. Disponível em: https://www.nejm.org/doi/ pdf/10.1056/NEJMp2006141? articleTools=true.

6. Mahase, E. Covid-19: most patients require mechanical ventilation in first 24 hours of critical care. $\mathrm{Br}$ Med J (Clin Res Ed). 24 de Março de 2020. doi: https://doi.org/10.1136/bmj.m1201.

7. Truog, R. D.; Mitchell, C.; Daley, G. Q. The Toughest Triage - Allocating Ventilators in a Pandemic. N Engl J Med [Internet]. 23 de Março de 2020. Disponível em: https://www.nejm.org/doi/full/10.1056/NEJMp2005689

8. Bhutta, Z. A.; Basnyat, B.; Saha, S.; Laxminarayan, R. Covid-19 risks and response in South Asia. The BMJ. 25 de Março de 2020. doi: https://doi.org/10.1136/bmj.m1190. 9. Pacheco, S. Ministério Saúde compra mais 3,3 mil respiradores fabricados no Brasil. Ministério da Saúde. 20 de Abril de 2020. Disponível em: https://www.saude. gov.br/noticias/agencia-saude/46759-ministerio-saudecovera-mais-3-3-mil-respiradores-fabricados-no-brasil

10. Rache, B.; Rocha, R.; Nunes, L.; Spinola, P.; Malik, A. M.; Massuda, A. Necessidades de Infraestrutura do SUS em Preparo à COVID-19: Leitos de UTI, Respiradores e Ocupação Hospitalar [Nota Técnica n.3]. IEPS: São Paulo. Março de 2020. Disponível em: https://ieps. org.br/wp-content/uploads/2020/04/IEPS-NT3.pdf.

11. Peng, F.; Tu, L.; Yang, Y.; Hu, P.; Wang, R.; Hu, Q.; Cao, F.; Jiang, T.; Sun, J.; Xu, G.; Chang, C. Management and Treatment of COVID-19: The Chinese Experience. Can J Cardiol. 17 de Abril de 2020. doi: 10.1016/j. 
cjca.2020.04.010. PubMed Central PMCID: PMC7162773.

12. World Health Organization. WHO Clinical management of severe acute respiratory infection (SARI) when COVID-19 disease is suspected. Who [Internet]. 13 de Março de 2020. Disponível em: https://www. who.int/publications-detail/clinicalmanagement-of-severe-acute-respiratory-infectionwhen-novel-coronavirus-(ncov)-infection-is-suspected.

13. Bhatraju, P. K.; Ghassemieh, B. J.; Nichols M.; Kim, R.; Jerome, K. R; Nalla, A. K; Greninger, A. L. et al. Covid-19 in Critically III Patients in the Seattle et al. Covid-19 in Critically III Patients in the Seattle Region - Case Series. N Engl J Med. 30 de Março
de2020. Disponível em: https://www. nejm.org/doi de2020. Disponível em: https://www.nejm.org/doi/ full/10.1056/NEJMoa2004500?url_ver=Z39.88-2003\&rfr_-

14. Ministério da Saúde. DATASUS Tecnologia da Informação a Serviço do SUS. CNES - Recursos Físicos Equipamentos - Brasil. RespiradorNentilador segundo Equipamentos - Brasil. Respirador/Nentilador segundo Região. Abril de 2020. Disponível em: http://tabnet.
datasus.gov.br/cgi/tabcgi.exe? cnes/cnv/equipobr.def datasus.gov.br/cgi/tabcgi.exe?cnes/cnv/equipobr.def

15. Ministério da Saúde. Diretrizes para o diagnóstico e tratamento da COVID-19. Secretaria de Ciência, Tecnologia, Inovação e Insumos Estratégicos em Saúde (SCTIE), v. 04. Brasília, 07 de Maio de 2020. Disponive em: https://portalarquivos.saude.gov.br/images/pdf/2020/ May/08/Diretriz-Covid19-v4-07-05.20h05m pdf

16. Meng, L.; Qiu, H.; Wan, L.; Ai, Y.; Xue, Z.; Guo, Q.; Deshpande, R.; Zhang, L.; Meng, J.; Tong, C.; Liu, H. Xiong, L. Intubation and Ventilation amid the COVID-19 Outbreak: Wuhan's Experience. Special section: doi: https://doi.org/10.1097/ALN.0000000000003296

17. Uhlig, S. Ventilation-induced lung injury and mechanotransduction: stretching it too far? Am J Physiol Lung Cell Mol Physiol. Maio de 2002. V. 282, n. 5, p. L892-L896. PubMed PMID: 11943651. doi: 10.1152/ajplung.00124.2001

18. Associação de Medicinalntensiva Brasileira(AMIB). Amib apresenta dados atualizados sobre leitos de UTI no Brasil. Março de 2020. São Paulo. Disponível em: http://www.epsjv. fiocruz.br/sites/default/files/files/dados_uti_amib(1).pdf
19. Associação de Medicina Intensiva Brasileira (AMIB). CENSO AMIB das UTIs Brasileiras: 2016. Disponivel em:

20. Ministério da Saúde. Resolução-RDC n 7, de 24 de fevereiro de 2010. Dispõe sobre os requisitos mínimos para funcionamento de Unidades de Terapia Intensiva e dá outras providências. Diário Oficial daUnião.24deFevereirode 2010.

21. Arie, S. Covid-19: Can France's ethical support units help doctors make challenging decisions? The BML. 02 de

22. Archard, D.; Caplan, A. Is it wrong to prioritise younger patients with covid-19? The BMJ. 22 de Abril de 2020. doi: https://doi.org/10.1136/bmj.m1509

23. Hick, J. L.; Biddinger, P. D. Novel Coronavirus and Old Lessons - Preparing the Health System for the Pandemic. N Engl J Med [Internet]. 25 de Março de 2020. Disponível em: https://www.nejm.org/doi/full/10.1056/NEJMp2005118

24. Bong, C. L.; Brasher, C; Chikumba, E; McDougall, R.; Mellin-Olsen, J.; Enright, A. The COVID-19 Pandemic: Effects on Low and Middle-Income Countries. Anesthanalg. 1 de Abril de 2020. doi: 10.1213/ANE.0000000000004846. PubMed PMID: 32243287. PubMed Central PMCID. PMC7173081.

25. Xie, J.; Tong, Z.; Guan, X.; Du, B.; Qiu, H.; Slutsky, A. S. Critical care crisis and some recommendations during the COVID-19epidemicinChina. IntensiveCareMed. 2 deMarço de 2020. doi: 10.1007/s00134-020-05979-7. PubMed PMID: 32123994. PubMed Central PMCID: PMC7080165.

26. Wells, C. R.; Fitzpatrick, M. C.; Sah, P.; Shoukat, A.; Pandey, $A_{i}$ El-Sayed, A, M et al. Projecting the demand for ventilats at the Peak of the COVID-19 outbeak in the USA. Lancet Infect Dis. 21 de Abril de 2020. doi: 10.1016/S1473-3099(20)30315-7. PubMed PMID: 32325039. PubMed Central PMCID: PMC7172723.

27. Elavarasan, M. R; Pugazhendhi, R. Restructured society and environment: A review on potential technological strategies to control the COVID-19 panderic SciTotal Environ.23 de Abril de 2020. do: panderic. SciTotal Environ.23 de A 32336562. PubMed Central PMCID: PMC7180041.
28. Clarke, A. L.; Stephens, A. F.; Liao, S; Byrne, T. J.; Gregory, S. D. Coping with COVID冈19 ventilator splitting with differential driving pressures using standard hospital equipment. Anaesthesia. 9 de Abril de 2020.

29. Anesthesia Patient Safety Foundation. Declaração conjunta sobre vários pacientes por ventilador. APSF. 26 de Março de 2020. Disponível em: $\quad$ https://www.apsf.org/news-updates/jointstatement-on-multiple-patients-per-ventilator/.

30. National Heart, Lung, and Blood Institute. Ventilator/ Ventilator Support. NHLBI. Disponível em: https://www. nhlbi.nih.gov/health-topics/ventilatorventilator-support. 